\title{
Changes in risk behaviours and prevalence of sexually transmitted infections following HIV preventive interventions among female sex workers in five districts in Karnataka state, south India
}

\author{
B M Ramesh, ${ }^{1,2}$ Tara S H Beattie, ${ }^{3}$ Isac Shajy, ${ }^{2}$ Reynold Washington, ${ }^{1,2,4}$ \\ Latta Jagannathan, ${ }^{5}$ Sushena Reza-Paul, ${ }^{1}$ James F Blanchard, ${ }^{1}$ Stephen Moses ${ }^{1,6}$
}

${ }^{1}$ Department of Community Health Sciences, University of Manitoba, Winnipeg, Canada ${ }^{2}$ Karnataka Health Promotion Trust, Bangalore, India ${ }^{3}$ Department of Epidemiology and Population Health, London School of Hygiene and Tropical Medicine, London, UK ${ }^{4}$ St John's Medical College and Hospital, Bangalore, India ${ }^{5}$ Bangalore Medical Services Trust, Bangalore, India ${ }^{6}$ Departments of Medical Microbiology and Medicine, University of Manitoba, Winnipeg, Canada

\section{Correspondence to} Dr B M Ramesh, Karnataka Health Promotion Trust, IT/BT Park, 5th Floor, No 1-4, Rajajinagar Industrial Area, Behind KSSIDC Administrative Office, Rajajinagar, Bangalore 560 044, India;

bmramesh@khpt.org

Accepted 26 October 2009

\section{UNLOCKA}

This paper is freely available online under the BMJ Journals unlocked scheme, see http://sti. bmi.com/site/about/unlocked. xhtml.

\begin{abstract}
Objectives To examine the impact of a large-scale HIV prevention programme for female sex workers (FSW) in Karnataka state, south India, on the prevalence of HIV/ sexually transmitted infections (STI), condom use and programme coverage.
\end{abstract}

Methods Baseline and follow-up integrated biological and behavioural surveys were conducted on random samples of FSW in five districts in Karnataka between 2004 and 2009.

Results 4712 FSW participated in the study (baseline 2312; follow-up 2400), with follow-up surveys conducted 28-37 months after baseline. By follow-up, over $85 \%$ of FSW reported contact by a peer educator and having visited a project STI clinic. Compared with baseline, there were reductions in the prevalence of HIV $(19.6 \%$ vs $16.4 \%$, adjusted odds ratio (AOR) $0.81,95 \% \mathrm{Cl} 0.67$ to $0.99, p=0.04)$; high-titre syphilis (5.9\% vs $3.4 \%$, AOR $0.53,95 \% \mathrm{Cl} 0.37$ to $0.77, \mathrm{p}=0.001$ ); and chlamydia and/ or gonorrhoea (8.9\% vs $7.0 \%$, AOR $0.72,95 \% \mathrm{Cl} 0.54$ to $0.94, p=0.02)$. Reported condom use at last sex increased significantly for repeat clients $(66.1 \%$ vs $84.1 \%$, AOR $1.98,95 \%$ Cl 1.58 to $2.48, \mathrm{p}<0.001$ ) and marginally for occasional clients $(82.9 \%$ vs $88.0 \%$, AOR $1.22,95 \% \mathrm{Cl}$ 0.89 to $1.66, p=0.2)$, but remained stable for regular partners (32\%). Compared with street and home-based FSW, brothel-based FSW were at highest risk of HIV and STI, despite high levels of reported condom use.

Conclusions This large-scale HIV prevention programme for FSW achieved reductions in HIV and STI prevalence, high rates of condom use with clients and high rates of programme coverage. Improved strategies to increase condom use with regular partners and reduce the vulnerability of brothel-based FSW to HIV are required.

India has an estimated 2.4 million people living with HIV. ${ }^{1}$ The HIV epidemic is highly heterogeneous, ${ }^{1}$ and the majority of HIV infections occur through heterosexual transmission, with unprotected paid sex a major transmission route, particularly in the southern states. ${ }^{2}$ There is little published evidence demonstrating the efficacy of female sex worker (FSW) preventive interventions, either in India ${ }^{3}{ }^{4}$ or elsewhere. ${ }^{5}$ Moreover, the processes required to bring about changes to FSW community norms in terms of practising safer sex and the treatment of sexually transmitted infections (STI) are unclear. ${ }^{67}$

Karnataka state in southern India has a relatively advanced HIV epidemic, with the adult HIV prevalence exceeding $1 \%$ for the past 13 years in several districts. $^{8} 9$ In 2003, the University of Manitoba (Canada), in partnership with the government of Karnataka, established the Karnataka Health Promotion Trust, providing focused HIV prevention programmes and services for highrisk groups in 18 districts across the state. The programme is funded by the Bill \& Melinda Gates Foundation under its India AIDS initiative, Avahan, ${ }^{10}$ and covers over 60000 FSW and 20000 men who have sex with men/transgender individuals. Targeted HIV prevention programmes and services have been rapidly scaled up, with the following main elements for FSW: (1) participatory mapping and enumeration exercises to estimate the number and typologies of FSW; (2) peer-mediated outreach to identify and address difficulties reported by FSW, plus behaviour change communication to promote condom use and regular STI screening; (3) establishment of dedicated sexual health services for FSW and their regular partners, offering STI identification and syndromic case management, routine speculum examination and periodic presumptive treatment for FSW every 3-6 months for chlamydia and gonorrhoea (1 g azithromycin and $400 \mathrm{mg}$ cefixime); recently, these services have been expanded to offer syphilis screening and treatment and HIV counselling and testing; (4) advocacy with key stakeholders, such as the police and local government officials and the establishment of 24-h crisis response teams to assist sex workers during crises; (5) creation of 'drop-in centres' to provide welcoming safe spaces, promote dialogue among FSW, and provide services such as community kitchens and literacy classes; and (6) community mobilisation and capacity building of communitybased organisations to implement HIV prevention programmes and services for their communities. ${ }^{11}$

To assess the impact of these intervention activities on programme coverage, condom use and STI and HIV prevalence among FSW over time, we conducted a series of anonymised, integrated behavioural and biological assessments (IBBA) with random samples of FSW in five districts across the state (Mysore, Belgaum, Shimoga, Bellary and Bangalore Urban). Previously, results have been presented for Mysore district only. ${ }^{6}$ In this paper we extend those findings, and present evidence of declining HIV and STI prevalence, high rates of condom use with clients and high rates of programme coverage. 


\section{METHODS}

\section{Study design and sampling}

The five IBBA districts were chosen purposively, based on Karnataka's sociocultural regions and the size of high-risk populations. Programmes were initiated in each district between January 2004 and April 2005, with baseline cross-sectional surveys conducted 7-19 months later. ${ }^{12}$ Follow-up cross-sectional surveys were conducted 28-37 months after the baseline surveys, ${ }^{12}$ and all surveys were conducted between 2004 and 2009.

Sample size calculations were designed to detect a $10-15 \%$ increase in condom use with $90 \%$ power and alpha error of $5 \%$, assuming a baseline value for consistent condom use with commercial clients of $50 \%$. It was thus estimated that 385 participants (rounded to 400) were required for each cross-sectional survey round. In Bangalore Urban, two sampling domains were utilised (street-based FSW and non-street-based FSW), with a target sample of 400 FSW in each domain for each round.

A probability-based sampling method was used. Conventional cluster sampling was used for FSW selling sex at home, or in brothels, lodges and dabhas, where the population of FSW was relatively stable. Conventional time-location cluster (TLC) sampling was used for street-based FSW, and a variation of the TLC sampling was used in the baseline survey in Mysore city, wherein the three sex work zones were divided into TLC and FSW were recruited proportionately from all the TLC. ${ }^{13}$ The same sampling technique was used for baseline and follow-up surveys.

\section{Behavioural questionnaire}

A behavioural questionnaire was designed to be culturally sensitive and context specific, and was adapted from previous surveys, ${ }^{14}$ as previously described. ${ }^{6}$ All interviews were conducted in Kannada (the local language) by trained researchers, except in the second round in Mysore, where trained FSW who were not programme staff conducted the interviews. To ensure confidentiality, the names of participants were not recorded and cannot be linked to the data.

\section{Laboratory methods}

Blood samples were taken to test for HIV and syphilis, and urine samples were taken to test for gonorrhoea and chlamydial infection, as previously described. ${ }^{6}$ All positive specimens and $10 \%$ of negative specimens were sent to the National AIDS Research Institute in Pune, India, for quality assurance. In Mysore district only, urine was tested for Trichomonas vaginalis, as previously described. ${ }^{6}$ Chlamydia and gonorrhoea tests conducted on samples from Bellary, Belgaum and Bangalore Urban used the Gen-Probe Aptima assay (Gen-Probe Inc, San Diego, California, USA), whereas tests on samples from Mysore and Shimoga used the Roche Amplicor system (Roche Molecular Diagnostics, Pleasanton, California, USA).

\section{Statistical analyses}

Data were double-entered using a Microsoft Access database (Microsoft, Redmond, Washington, USA). All statistical analyses were performed using the survey data analysis techniques in STATA, version 10.0. Appropriate weights were used to account for the differential recruitment of FSW by typology within districts, differential non-response rates and differential probabilities of FSW selection across districts. Odds ratios were used as the measure of association, and the Wald $\chi^{2}$ test was the statistical test used. There were few missing data in either round, except for syphilis in 36 participants at the baseline survey in Mysore. Women who participated in both rounds were included in all analyses, as due to the unlinked, anonymised nature of the surveys, it was not possible to know which individuals in the first round also participated at follow-up. Primary outcomes were STI/HIV prevalence, reported condom use and exposure to the HIV intervention programme components. In the multivariate models, the sociodemographic variables that were significantly different in the two surveys were adjusted for, as shown in the tables.

\section{RESULTS \\ Study population}

A total of 2312 FSW participated in the baseline surveys, and 2400 participated at follow-up surveys; 399 women (16.6\%) reported taking part at both time points. The median age of FSW was 30 years (interquartile range (IOR) 19-41) and the majority $(67 \%)$ were illiterate (table 1$)$. Six per cent of the study population were devadasis, a group of FSW in northern Karnataka who are part of a long-standing tradition in which adolescent girls are dedicated to gods and goddesses and are subsequently inducted into sex work.

FSW reported a median age of sexual debut of 15 years (IOR 11-19), with participants starting to sell sex at a median of 25 years (IOR 15-35). The most frequent places of solicitation of sex were public places (55\%) and at home (35\%), with a minority $(10 \%)$ brothel, lodge or dabha-based (hereafter briefly labelled 'brothel-based'). Approximately $80 \%$ of participants reported selling sex for more than 2 years at both survey rounds. Between baseline and follow-up, the mean charge per sex act increased substantially, so that by the follow-up survey, weekly earnings from sex work had nearly doubled (mean weekly earnings baseline 1632 Indian rupees (US\$33) vs follow-up 2915 rupees (US \$60) $(\mathrm{p}<0.001)$ (table 1).

\section{Exposure to the intervention programme}

Despite high levels of programme exposure reported at baseline (the baseline surveys were conducted 7-19 months after programme initiation and thus were not 'true' baselines), there were large increases $(p<0.001)$ in the proportions of FSW who had been exposed to almost all components of the intervention programme by the time of the follow-up surveys (table 2). Therefore, by follow-up, virtually all FSW (95.3\%) had been visited by a peer educator, $76.6 \%$ had visited the drop-in centre, $85.1 \%$ had visited the project sexual health clinic and $63.6 \%$ had received the 'grey pack', for presumptive treatment of chlamydia and gonorrhoea (table 2). Furthermore, the proportion of FSW who reported having seen a condom demonstration increased from $73.3 \%$ to $89.0 \%$, and the proportion reporting condom breakage in the previous month fell from $17.0 \%$ to $14.1 \%$ (table 2 ).

When we stratified FSW according to their reported duration of exposure to the HIV intervention programme (not exposed, $<12$ months, $12-23$ months, $24-33$ months, $>33$ months), the proportion of FSW who reported accessing various components of the intervention programme increased significantly with increasing duration of exposure to the programme (figure 1a).

\section{Condom use}

At the baseline surveys, levels of reported condom use with clients were already high, with over $65 \%$ reporting using a condom at last sex with their last occasional or repeat client (table 3). Reported condom use with regular partners was much lower, with one-third (32\%) reporting using a condom at last sex.

Compared with baseline, in both univariate and multivariate analyses, there was a substantial increase in the proportion of FSW reporting zero unprotected sex acts in the past month with 
Table 1 Sociodemographic and sex work characteristics of FSW at baseline and follow-up surveys

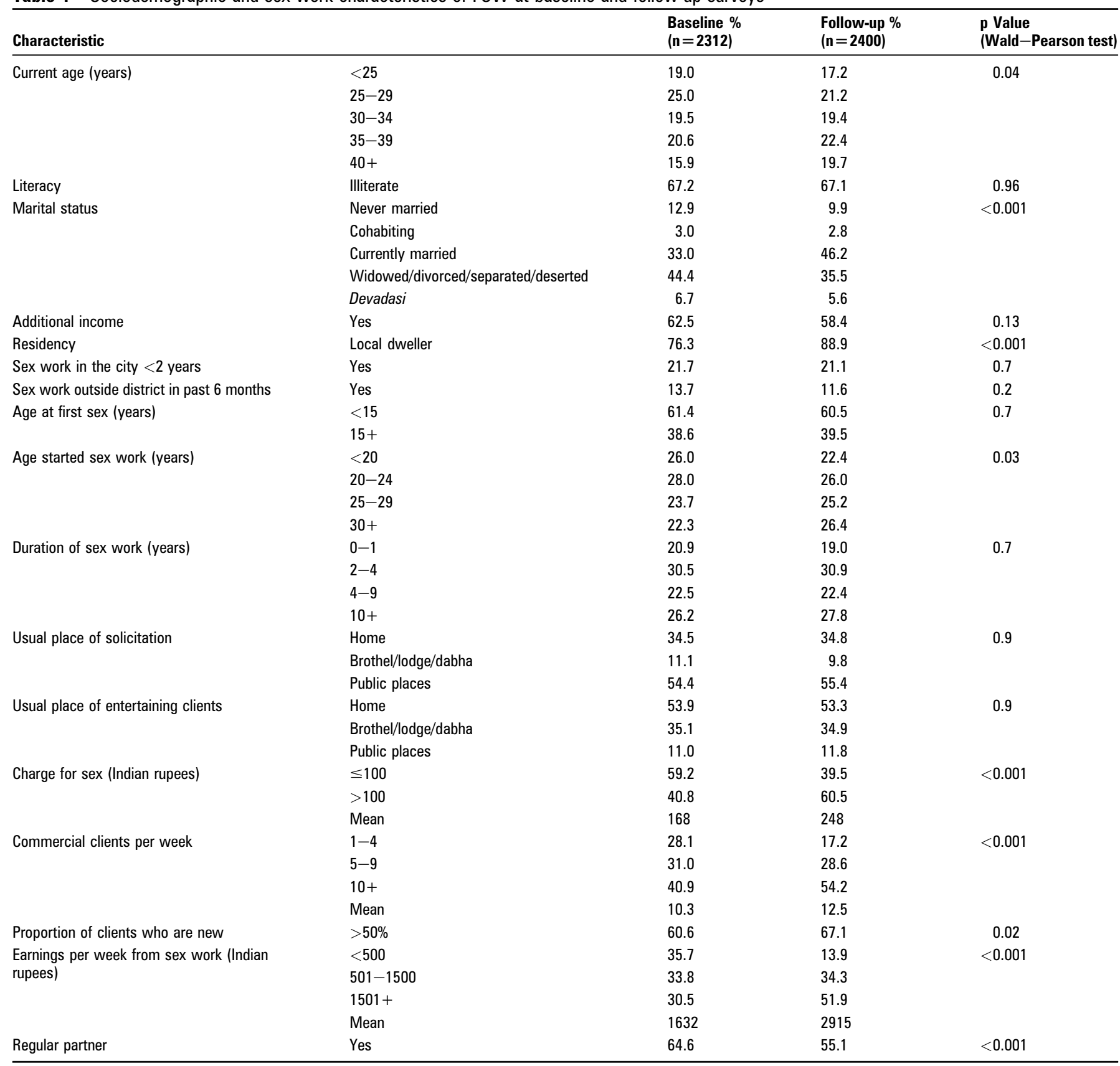

FSW, female sex worker.

their commercial clients (table 3). Although the proportion of FSW reporting condom use at last sex with occasional clients increased only marginally from $82.9 \%$ to $88.0 \%$, reported condom use at last sex with repeat commercial clients increased substantially from $66.1 \%$ to $84.1 \%$. Reported condom use at last sex with regular partners remained stable (table 3 ).

When FSW were stratified according to their reported length of exposure to the HIV prevention programme, there were striking increases with increasing duration of programme exposure $(p<0.01)$ in all measures of reported condom use, with both commercial clients and regular partners (figure $1 b$ ).

\section{STI prevalence}

HIV prevalence was high among this population, with one-fifth of FSW testing HIV-1 seropositive at baseline (table 3). HIV prevalence was highest in Belgaum (33.9\%) and Mysore (26.1\%), with lower rates seen in Bellary (15.7\%), Bangalore Urban $(12.7 \%)$ and Shimoga (9.7\%). Baseline prevalence of bacterial STI was relatively low for a sex worker population, with $10 \%$ of participants testing positive for syphilis, $7 \%$ for chlamydia and $4 \%$ for gonorrhoea. Approximately $6 \%$ had high-titre syphilis, indicative of more recent or active syphilis infection (table 3 ).

At the follow-up survey, HIV prevalence fell significantly, from $19.6 \%$ to $16.4 \%$ (table 3 ), with reductions seen in all districts (data not shown). There were also reductions in the prevalence of any syphilis infection and of high-titre syphilis (table 3). Despite low baseline rates, the proportion infected with either chlamydia and/or gonorrhoea also fell significantly (table 3).

In both univariate and multivariate analyses, reductions in chlamydia and/or gonorrhoea infection were strongly $(p<0.001)$ associated with peer education, visiting the project sexual health clinic and receiving the 'grey pack', but there were no significant 
Table 2 Univariate and multivariate analyses of programme exposure and intermediate causal characteristics among FSW at follow-up compared with baseline

\begin{tabular}{lllll}
\hline Programme exposure & $\begin{array}{l}\text { Baseline \% } \\
\text { (n=2312) }\end{array}$ & $\begin{array}{l}\text { Follow-up \% } \\
\text { (n=2400) }\end{array}$ & Crude 0R (95\% CI) & $\begin{array}{l}\text { Adjusted OR (95\% CI) } \\
\text { adjusted OR }\end{array}$ \\
\hline Ever visited by a peer educator & 82.8 & 95.3 & $4.25(2.81$ to 6.43$)$ & $4.35(3.14$ to 6.01$)$ \\
Ever visited the drop-in centre & 32.2 & 76.6 & $6.88(4.79$ to 9.87$)$ & $8.72(7.01$ to 10.84$)$ \\
Ever visited the project sexual health clinic & 68.4 & 85.1 & $2.63(2.00$ to 3.45$)$ & $2.77(2.14$ to 3.59$)$ \\
Ever received 'grey pack' & 58.4 & 63.6 & $1.24(1.00$ to 1.55$)$ & $1.28(0.98$ to 1.67$)$ \\
Ever witnessed a condom demonstration & 73.3 & 89.0 & $2.93(1.87$ to 4.58$)$ & $2.97(2.14$ to 4.13$)$ \\
Condom breakage in the past month & 17.0 & 14.1 & $0.80(0.58$ to 1.11$)$ & $0.75(0.60$ to 0.92$)$
\end{tabular}

Multivariate models were adjusted for the following variables: district, age, marital status, residency status, usual place of solicitation, age started sex work, charge per sex act, weekly sex work income, proportion of clients who were new and proportion of female sex workers (FSW) with regular partners.

associations between reductions in high-titre syphilis and exposure to any programme component (data not shown). Furthermore, there were no significant associations between reductions in any curable STI and reported condom use (data not shown).

In Mysore district only, FSW were also tested for trichomonas infection in both rounds (data not shown). ${ }^{6}$ Almost one-third $(32.9 \%)$ were infected at baseline, but this proportion fell to
$13.5 \%$ by the follow-up survey (adjusted odds ratio $0.3,95 \% \mathrm{CI}$ 0.2 to $0.4, \mathrm{p}<0.001$ )

When we stratified FSW according to their duration of exposure to the HIV intervention programme (figure 1c), although there were no clear trends for syphilis, rates of infection with chlamydia and/or gonorrhoea fell with increasing programme exposure, from $13 \%$ among FSW who had not been exposed to
Figure 1 Condom use and HIV/ sexually transmitted infection prevalence according to the duration of exposure to the HIV intervention programme. (a) Exposure to programme components and duration of programme exposure. (b) Condom use and duration of programme exposure. (c) HIV/STI prevalence and duration of programme exposure. ${ }^{*} \mathrm{p}<0.05,{ }^{*} \mathrm{p}<0.01$.

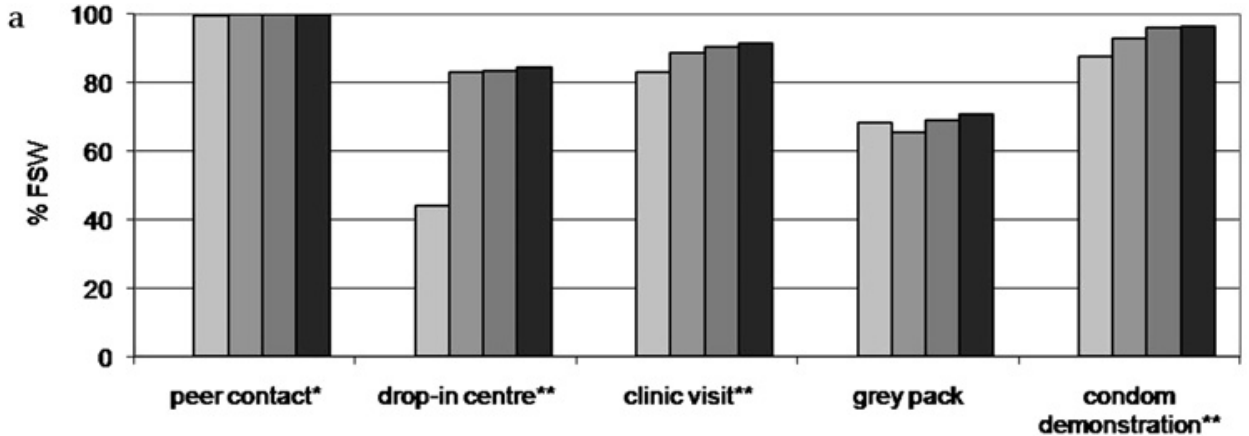

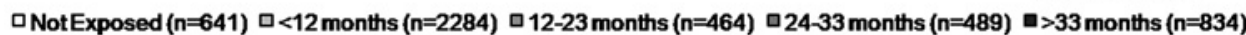

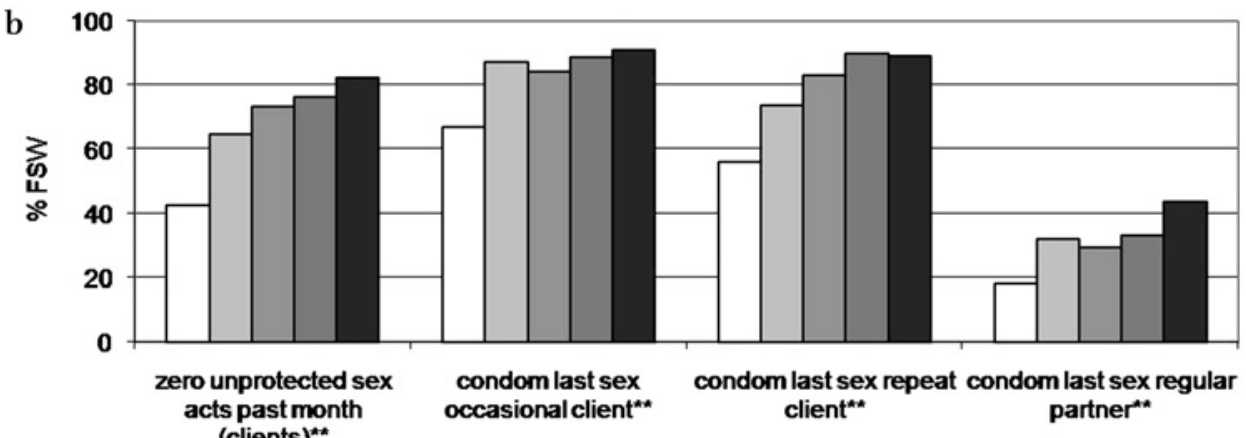
(clients) ${ }^{\text {t* }}$

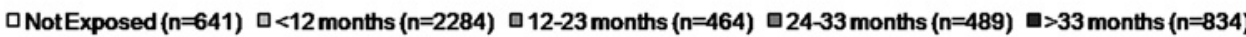

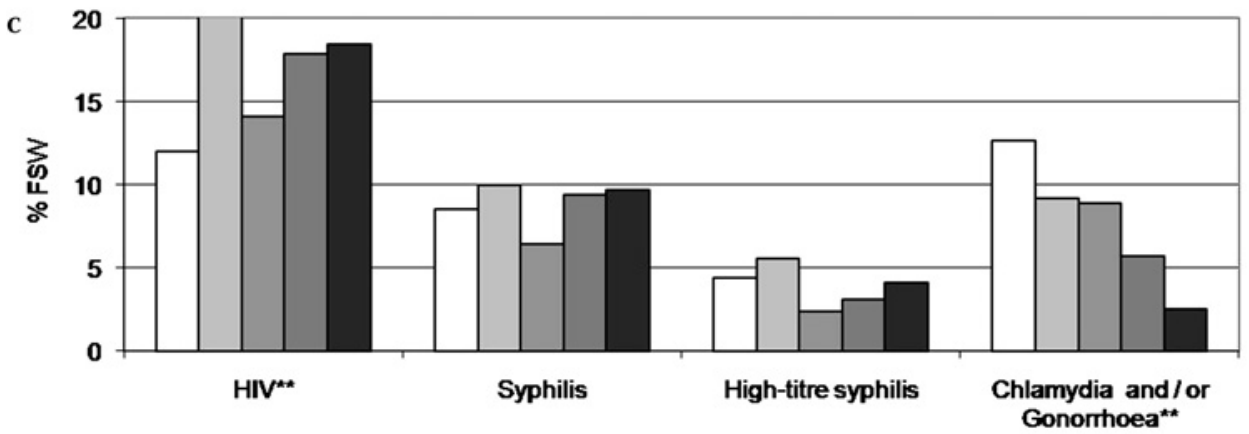

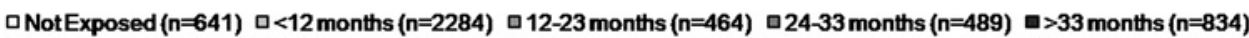


Table 3 Univariate and multivariate analyses of condom-related outcomes and STI prevalence among FSW at follow-up compared with baseline

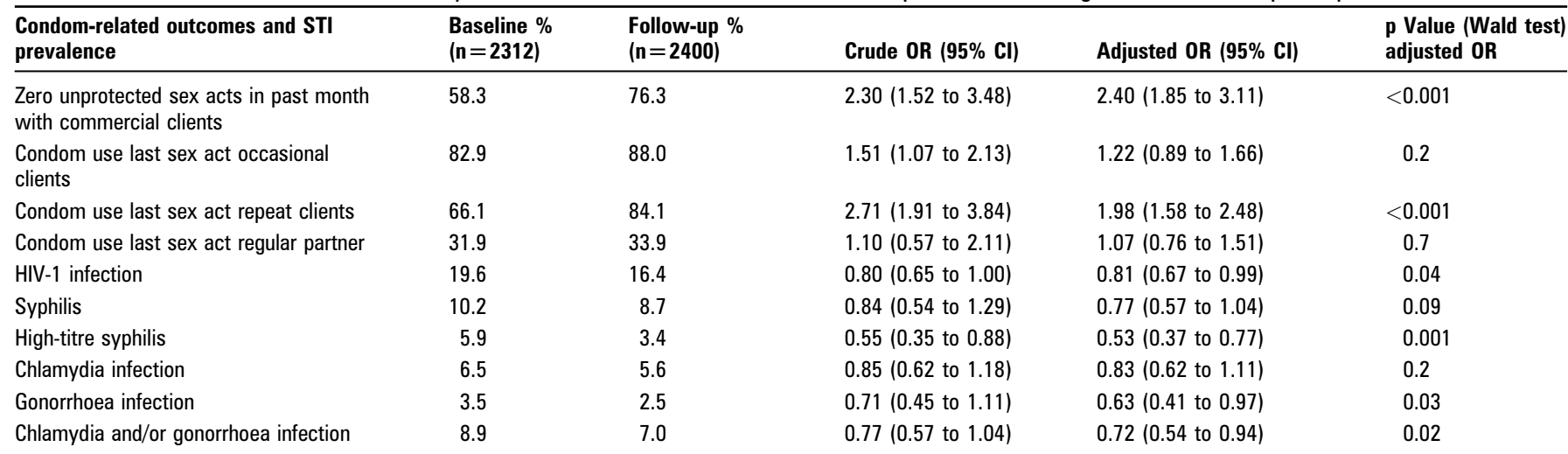

Multivariate models were adjusted for the following variables: district, age, marital status, residency status, usual place of solicitation, age started sex work, charge per sex act, weekly sex work income, proportion of clients who were new and proportion of female sex workers (FSW) with regular partners.

STI, sexually transmitted infection.

the HIV intervention programme to 3\% among FSW exposed for more than 33 months $(p<0.001)$.

\section{FSW typology}

HIV prevalence was substantially higher among brothel-based FSW compared with home and street-based FSW, with more than a quarter of brothel-based FSW testing HIV seropositive (table 4). Similarly, infection with gonorrhoea and/or chlamydia was highest in this group, at $12 \%$ (table 4). Although the prevalences of curable STI decreased among street-based FSW between the two surveys, those among brothel and home-based FSW remained stable (table 4).

Despite these high HIV and STI rates, brothel-based FSW reported higher condom use with their sexual partners, compared with the other typologies (table 4). However, by the follow-up survey, they were less likely to have previously visited the project drop-in centre or sexual health clinic, or to have received a 'grey pack', compared with FSW who solicited sex at home or in a public place. Moreover, brothel-based FSW were likely to be younger (mean age 22.6 years brothel-based vs 24.8 years home-based vs 25.1 years street-based, $p<0.001$ ), to charge less per sex act (mean charge 154 rupees brothel-based vs 195 rupees home-based vs 227 rupees street-based, $p<0.001$ ), and to entertain double the number of clients per week (mean clients per week 22.3 brothel-based vs 9.4 home-based vs 10.7 streetbased FSW, $p<0.001$ ), compared with home and street-based sex workers. However, despite charging less per sex act, they were likely to earn more per week from sex work compared with the other typologies because of their higher client volume (mean earnings per week 2637 rupees vs 1837 rupees vs 2481 rupees for brothel vs home vs street-based FSW, respectively, $\mathrm{p}<0.001)$. In addition, they were more likely to have previously sold sex in Mumbai ( $6.3 \%$ brothel vs $1.7 \%$ home vs $2.6 \%$ street-based FSW, respectively, $\mathrm{p}=0.008)$.

\section{DISCUSSION}

This is among the first studies to evaluate the effect on HIV and STI rates of a preventive intervention that combines risk reduction, condom promotion and improved access to sexual health services with empowerment of sex workers. ${ }^{5}{ }^{15-17}$ Unlike other FSW populations in India, which have reported sharp increases in HIV prevalence over time, ${ }^{7}$ our study found significant reductions in the prevalences of HIV, high-titre syphilis, chlamydial infection and gonorrhoea among participants between baseline and follow-up surveys. Furthermore, there were extremely high levels of reported programme coverage and high levels of reported condom use with commercial clients.

\section{HIV/STI rates}

As the cross-sectional surveys were unlinked and anonymised, it is not possible to ascertain what the rate of new HIV infection acquisition was during the study period. However, the significant reductions in HIV and STI prevalence suggest that sexual health promotion programmes and services are now starting to reach this population, and safer sex practices are being adopted. As infections with STI have been shown to increase the likelihood of HIV transmission, ${ }^{19-21}$ it is expected that the reductions in STI prevalence observed among FSW in our study, in conjunction with increased condom use, may have contributed to reductions in new HIV transmission events. ${ }^{2-24}$ Concerted efforts were made during programme initiation to ensure accessible, user-friendly STI services for this population, including active involvement of the FSW community in the recruitment of clinical staff and the selection of STI clinic locations. The data suggest that these efforts may have translated into reduced chlamydia and gonorrhoea prevalence, through more rapid detection and treatment of infections. Rates of high-titre syphilis, but not all syphilis infection, also fell significantly between baseline and follow-up. With the exception of Mysore, services for screening and treatment of syphilis infection at the project clinics in the other districts only started to take off in 2008. With enhanced and expanded syphilis testing and treatment facilities in place, we expect syphilis rates to fall by the next crosssectional survey round. ${ }^{25}$

\section{Reported condom use}

Several studies among both FSW and other populations have reported increases in condom use following HIV preventive intervention programmes, ${ }^{5}{ }^{26}$ and by the time of the follow-up surveys in our populations, over $85 \%$ of FSW reported using a condom at last sex with commercial clients. However, because of the very high levels of condom use reported with clients at baseline, only condom use at last sex with repeat clients had significantly increased by the follow-up survey round. Baseline surveys were conducted $8-16$ months after programme initiation, by which time levels of condom use with occasional clients were already very high, not leaving much room for an increase; unfortunately, it is difficult to undertake cross-sectional surveys among sex worker communities until prevention programmes are underway and trust with the community is well established. However, before the start of the intervention programme, and 
Table 4 Univariate and multivariate analyses of STI prevalence, condom use and programme exposure at baseline and follow-up, according to usual place of solicitation

\begin{tabular}{|c|c|c|c|c|}
\hline & & $\begin{array}{l}\text { Home ( } \mathrm{N}=1652 \text { ) } \\
\text { (baseline/follow-up) }\end{array}$ & $\begin{array}{l}\text { Brothel/lodge/Dabha } \\
\text { (n=474) (baseline/ } \\
\text { follow-up) }\end{array}$ & $\begin{array}{l}\text { Street/public places } \\
\text { ( } n=2586 \text { ) (baseline/ } \\
\text { follow-up) }\end{array}$ \\
\hline \multirow[t]{3}{*}{ HIV prevalence } & $\%$ & $13.5 / 11.8$ & $33.2 / 26.1$ & 21.3/17.6 \\
\hline & Crude OR (95\% CI) & $0.85(0.58$ to 1.26$)$ & $0.71(0.41$ to 1.23$)$ & $0.79(0.60$ to 1.05$)$ \\
\hline & Adjusted OR (95\% Cl) & $0.98(0.60$ to 1.58$)$ & $0.77(0.39$ to 1.52$)$ & $0.77(0.55$ to 1.01$)$ \\
\hline \multirow[t]{3}{*}{ High-titre syphilis } & $\%$ & $2.1 / 2.7$ & $3.8 / 2.3$ & $8.8 / 4.0$ \\
\hline & Crude OR (95\% Cl) & $1.31(0.60$ to 2.84$)$ & $0.60(0.13$ to 2.74$)$ & $0.43^{*}(0.27$ to 0.68$)$ \\
\hline & Adjusted OR (95\% CI) & $1.12(0.40$ to 3.13$)$ & $0.55(0.16$ to 1.88$)$ & $0.30 *(0.19$ to 0.49$)$ \\
\hline \multirow[t]{3}{*}{ Chlamydia and/or gonorrhoea infection } & $\%$ & $6.2 / 5.7$ & $12.2 / 12.2$ & $10.1 / 6.9$ \\
\hline & Crude OR (95\% Cl) & $0.92(0.55$ to 1.53$)$ & $0.99(0.39$ to 2.53$)$ & $0.66 \dagger(0.46$ to 0.94$)$ \\
\hline & Adjusted OR (95\% Cl) & $0.92(0.50$ to 1.69$)$ & $1.18(0.51$ to 2.69$)$ & $0.57^{*}(0.38$ to 0.87$)$ \\
\hline \multirow{3}{*}{$\begin{array}{l}\text { Condom use at last sex with commercial } \\
\text { client }\end{array}$} & $\%$ & $75.8 / 84.5$ & $98.0 / 95.6$ & $84.3 / 88.7$ \\
\hline & Crude OR (95\% Cl) & $1.74^{*}(1.24$ to 2.45$)$ & $0.49(0.15$ to 1.56$)$ & $1.47(0.89$ to 2.43$)$ \\
\hline & Adjusted OR (95\% CI) & $0.81(0.51$ to 1.29$)$ & $0.63(0.22$ to 1.80$)$ & $0.98(0.56$ to 1.71$)$ \\
\hline \multirow[t]{3}{*}{ Condom use at last sex with repeat client } & $\%$ & $62.2 / 77.4$ & $81.8 / 94.5$ & $64.6 / 79.7$ \\
\hline & Crude OR (95\% CI) & $2.08 *(1.48$ to 2.93$)$ & $3.82 \dagger(1.42$ to 10.26$)$ & $2.15^{*}(1.49$ to 3.10$)$ \\
\hline & Adjusted OR (95\% CI) & $1.67 \dagger(1.03$ to 2.71$)$ & $5.82^{*}(1.67$ to 20.28$)$ & $2.02^{*}(1.45$ to 2.81$)$ \\
\hline \multirow{3}{*}{$\begin{array}{l}\text { Condom use at last sex with regular } \\
\text { partner }\end{array}$} & $\%$ & $44.0 / 32.8$ & $42.7 / 37.5$ & $23.5 / 34.3$ \\
\hline & Crude OR (95\% CI) & $0.62 \dagger(0.40$ to 0.97$)$ & $0.81(0.27$ to 2.45$)$ & 1.70 (0.77 to 3.78$)$ \\
\hline & Adjusted OR (95\% CI) & $0.78(0.42$ to 1.45$)$ & $2.55(0.58$ to 11.16$)$ & $2.11 \dagger(1.00$ to 4.46$)$ \\
\hline \multirow[t]{3}{*}{ Ever visited by a peer educator } & $\%$ & $81.7 / 95.8$ & $90.9 / 94.3$ & $81.8 / 95.2$ \\
\hline & Crude OR (95\% CI) & $5.13^{*}(2.77$ to 9.48$)$ & $1.65(0.71$ to 3.82$)$ & $4.43^{*}(2.72$ to 7.21$)$ \\
\hline & Adjusted OR (95\% CI) & $6.95^{*}(4.21$ to 11.46$)$ & $4.59 *(1.91$ to 11.02$)$ & $3.73^{*}(2.44$ to 5.71$)$ \\
\hline \multirow[t]{3}{*}{ Ever attended the drop-in centre } & $\%$ & $26.4 / 73.7$ & $30.0 / 62.3$ & $36.3 / 80.9$ \\
\hline & Crude OR (95\% CI) & $7.78^{*}$ (5.16 to 11.72$)$ & $3.84^{*}(2.07$ to 7.13$)$ & $7.44^{*}(4.39$ to 12.60$)$ \\
\hline & Adjusted OR (95\% Cl) & $10.46^{*}(7.12$ to 15.38$)$ & $13.46^{*}$ (5.97 to 30.32$)$ & $8.82^{*}(6.70$ to 11.62$)$ \\
\hline \multirow[t]{3}{*}{ Ever attended the clinic } & $\%$ & $66.2 / 84.5$ & $76.7 / 74.7$ & $68.2 / 87.2$ \\
\hline & Crude OR (95\% CI) & $2.80 *(1.86$ to 4.20$)$ & $0.90(0.50$ to 1.63$)$ & $3.18 *(2.24$ to 4.53$)$ \\
\hline & Adjusted OR (95\% CI) & $3.43^{*}(2.23$ to 5.27$)$ & 1.36 (0.72 to 2.57$)$ & $2.92^{*}(2.07$ to 4.12$)$ \\
\hline \multirow[t]{3}{*}{ Ever received a 'grey pack' } & $\%$ & $53.2 / 58.5$ & $73.6 / 54.6$ & $58.6 / 68.4$ \\
\hline & Crude OR (95\% CI) & $1.24(0.91$ to 1.70$)$ & $0.43^{*}(0.25$ to 0.75$)$ & $1.53^{*}(1.16$ to 2.01$)$ \\
\hline & Adjusted OR (95\% CI) & $1.69 *(1.18$ to 2.42$)$ & $0.47 \dagger(0.26$ to 0.85$)$ & $1.33(0.94$ to 1.90$)$ \\
\hline
\end{tabular}

${ }^{*} \mathrm{p}<0.01$.

$\dagger p<0.05$.

Multivariate models were adjusted for the following variables: district, age, marital status, residency status, age started sex work, charge per sex act, weekly sex work income, proportion of clients who were new and proportion of female sex workers with regular partners. In addition, the first six rows were adjusted for: ever visited by a peer educator, ever attended the drop in centre, ever attended the clinic and ever received a 'grey pack'.

Odds ratios (OR) are in relation to follow-up versus baseline parameters.

STI, sexually transmitted infection.

under the most plausible scenarios of condom availability, it has been estimated elsewhere that there would only have been sufficient condoms available to cover $16-24 \%$ of all FSW sex acts, (JE Bradley, S Moses, J Blanchard et al, 2009, personal communication) suggesting condom use must have been quite low before programme initiation. In addition, special behavioural (qualitative) surveys conducted with FSW found that less than $20 \%$ of those interviewed reported condom use with their sexual partners before the intervention (Karnataka Health Promotion Trust and Centre Hospitalier Affilié Universitaire de Québec, 2009, unpublished data). The substantial increases observed in reported condom use with commercial clients with increasing duration of programme exposure suggests that exposure to the HIV intervention programme was enabling more condom use. Only one-third of FSW reported condom use at last sex with their regular partner (with whom negotiating condom use is more challenging), ${ }^{27}$ but we again found incremental increases in reported condom use with regular partners with increasing duration of programme exposure. Overreporting of condom use due to social desirability bias may partly explain the associations with condom use and programme exposure. However, it is likely that community mobilisation, empowerment of the sex work community and the embedding of HIV intervention programmes within an enabling environment has facilitated the ability of FSW to use condoms with their various sexual partners. ${ }^{15}$

\section{Empowerment of sex workers}

Although we did not directly measure the empowerment of sex workers, the high response rates, coupled with the extremely high rates of programme exposure by the second survey, and the sharp increase in the amount of money charged per sex act, suggest effective community mobilisation. The conversion of a hostile environment to a more facilitative enabling environment for sex work has been reported elsewhere as a contributing factor to improved condom use, ${ }^{15} 29$ and although that process was a key component of this programme, ${ }^{6}$ the programme exposure variables measured did not fully capture the complexities involved with risk reduction behaviours. We plan to include indicators related to empowerment, community mobilisation and the enabling environment in subsequent surveys.

\section{Sex work typology}

We found striking differences between place of solicitation and HIV prevalence, with more than a quarter of brothel-based FSW HIV seropositive, compared with $19 \%$ of street and $12 \%$ of 
home-based FSW. Rates of peer educator contact were similar across typologies, but brothel-based FSW were less likely to have visited the project sexual health clinic or received a 'grey pack' than home or street-based FSW, and this may have contributed to the higher rates of gonorrhoea and chlamydia also seen in this group. Moreover, brothel-based FSW were more likely to report having previously sold sex in Mumbai, where HIV prevalence rates among FSW have reached over $50 \% .{ }^{30}$ Although brothelbased FSW were more likely to report condom use with sexual partners, their client volume was also higher than among other typologies, putting them at greater risk of exposure to HIV/STI infection. Concerted efforts that target the most vulnerable women must continue and be strengthened, and this includes facilitating regular visits to healthcare providers, as well as promoting risk-reduction behaviours such as reducing high client volumes and promoting condom use with all sexual partners.

\section{Study limitations}

This study comprised a series of cross-sectional surveys, rather than a randomised, controlled trial design, and without a suitable control group, it is not possible to rule out that the results may have occurred independently of the intervention, instead reflecting natural trends in HIV or STI prevalence. However, the large sample size and the magnitude of the observed declines in STI rates make this exceedingly unlikely. In addition, the strong linear associations seen between the increasing duration of exposure to the HIV intervention programme, and both increased condom use and reduced high-titre syphilis, gonorrhoea and chlamydia prevalence, further supports the likelihood of a true programme effect. Social desirability bias in face-to face interviews such as these could have overestimated the rates of condom use and programme exposure. ${ }^{31}$ However, a major advantage of this study was the inclusion of biological data, which does not rely on self-reported behaviours. A further limitation is that the baseline surveys were conducted 7-19 months after programme initiation, and thus the magnitude of changes in condom use and STI rates may have been underestimated.

\section{Conclusions}

In conclusion, the results of this study suggest that a comprehensive HIV prevention programme among FSW can have a positive impact in terms of increased condom use and decreased STI and HIV prevalence. Improved strategies are required to increase condom use with regular partners and reduce brothel-based FSW vulnerability. STI and HIV prevention and control programmes should be scaled up in similar settings across India and the subcontinent.

\section{Key messages}

- This study evaluates the effect of an HIV preventive intervention that combines sexual risk reduction and improved access to sexual health services with empowerment of sex workers.

- Following baseline and follow-up surveys, substantial reductions were seen in the prevalences of HIV infection, high-titre syphilis and gonorrhoea/chlamydia.

- Programme coverage was extremely high, and substantial increases were seen in reported condom use with commercial clients, but not with regular partners.

- Brothel-based sex workers remain particularly vulnerable to HIV and STI.
Contributors BMR designed and supervised the cross-sectional, integrated behavioural and biological assessment (IBBA) surveys, performed analyses and contributed to writing the paper. TSHB performed analyses and wrote the first draft of the paper. SI co-designed and supervised the IBBA surveys and contributed to writing the paper. RW designed and supervised the implementation of the intervention programme and contributed to writing the paper. LJ supervised the laboratory testing. SR-P supervised the IBBA surveys and the implementation of the intervention programme, and contributed to writing the paper. JFB and SM designed the study and contributed to writing the paper.

Funding This research was funded by the Bill \& Melinda Gates Foundation. The views expressed herein are those of the authors and do not necessarily reflect the official policy or position of the Bill \& Melinda Gates Foundation.

\section{Competing interests None.}

Ethical approval Statutory approval for the conduct of the IBBA and their protocols was obtained by the Government of India's Health Ministry Screening Committee. The study was approved by the Institutional Ethical Review Board of St John's Medical College, Bangalore, India and the Health Research Ethics Board of the University of Manitoba, Winnipeg, Canada

Provenance and peer review Not commissioned; externally peer reviewed.

\section{REFERENCES}

1. UNAIDS. Asia: AIDS epidemic summary: regional summary. http://data.unaids.org/ pub/Report/2008/jc1527_epibriefs_asia_en.pdf, 2008 (Accessed 7 April 2009).

2. Halli SS, Blanchard J, Satihal DG, et al. Migration and HIV transmission in rural South India: an ethnographic study. Cult Health Sex 2007; 9:85-94.

3. Gangopadhyay DN, Chanda M, Sarkar K, et al. Evaluation of sexually transmitted diseases/human immunodeficiency virus intervention programs for sex workers in Calcutta, India. Sex Transm Dis 2005;32:680-4.

4. Fung IC, Guinness L, Vickerman $P$, et al. Modelling the impact and cost-effectiveness of the HIV intervention programme amongst commercial sex workers in Ahmedabad Gujarat, India. BMC Public Health 2007; 7:195.

5. Foss AM, Hossain M, Vickerman PT, et al. A systematic review of published evidence on intervention impact on condom use in sub-Saharan Africa and Asia. Sex Transm Infect 2007:83:510-16.

6. Reza-Paul S, Beattie T, Syed HU, et al. Declines in risk behaviour and sexually transmitted infection prevalence following a community-led HIV preventive intervention among female sex workers in Mysore, India. AIDS 2008;22(Suppl. 5): S91-100.

7. Basu I, Jana S, Rotheram-Borus MJ, et al. HIV prevention among sex workers in India. J Acquir Immune Defic Syndr 2004;36:845-52.

8. Population Foundation of India KSAPS, India-Canada Collaborative HIV/AIDS Project and Population Reference Bureau. HIVIAIDS in Karnataka: situation and response 2004. Bangalore: Karnataka State AIDS Prevention Society. http://www.prb.org/ pdf04/HIVinKarnataka.pdf (Accessed 7 April 2009).

9. Ramesh BM, Moses S, Washington R, et al. Determinants of HIV prevalence among female sex workers in four south Indian states: analysis of cross-sectional surveys in twenty-three districts. AIDS 2008;22 (Suppl. 5):S35-44.

10. Avahan. Avahan - the India AIDS initiative: the business of HIV prevention at scale New Delhi: The Bill \& Melinda Gates Foundation, 2008.

11. Gurnani V, Mallika A, Isac S, et al. Addressing violence against female sex workers in the context of HIV prevention programming: experience from Karnataka, South India. XVIIth International AIDS Conference. 3-8 August 2008. Abstract No WEPE0446. Mexico City, Mexico, 2008. http://www.aids2008.org/mainpage. $a s p x ?$ pageld =8

12. Ramesh B, Beattie T, Isac S, et al. Changes in risk behaviours and STI prevalence following community-led HIV preventive interventions among female sex workers in four districts in Karnataka State. Bangalore, India: Karnataka Health Promotion Trust and The University of Manitoba, 2009.

13. Magnani R, Sabin K, Saidel T, et al. Review of sampling hard-to-reach and hidden populations for HIV surveillance. AIDS 2005;19(Suppl. 2):S67-72.

14. Saidel T, Adhikary R, Mainkar M, et al. Baseline integrated behavioural and biological assessment among most at-risk populations in six high-prevalence states of India: design and implementation challenges. AIDS 2008;22(Suppl. 5): S17-34

15. Jana S, Basu I, Rotheram-Borus MJ, et al. The Sonagachi Project: a sustainable community intervention program. AIDS Educ Prev 2004;16:405-14.

16. Kerrigan D, Moreno L, Rosario $S$, et al. Environmental-structural interventions to reduce HIV/STI risk among female sex workers in the Dominican Republic. Am J Public Health 2006;96:120-5.

17. Shahmanesh M, Patel V, Mabey D, et al. Effectiveness of interventions for the prevention of HIV and other sexually transmitted infections in female sex workers in resource poor setting: a systematic review. Trop Med Int Health 2008;13:659-79

18. Bhave G, Lindan CP, Hudes ES, et al. Impact of an intervention on HIV, sexually transmitted diseases, and condom use among sex workers in Bombay, India. AIDS 1995;9(Suppl 1):S21-30.

19. Cohen MS, Hoffman IF, Royce RA, et al. Reduction of concentration of HIV-1 in semen after treatment of urethritis: implications for prevention of sexual transmission of HIV-1. AIDSCAP Malawi Research Group. Lancet 1997;349:1868-73. 
20. Kaul R, Kimani J, Nagelkerke NJ, et al. Risk factors for genital ulcerations in Kenyan sex workers. The role of human immunodeficiency virus type 1 infection. Sex Transm Dis 1997; 24:387-92.

21. Plummer FA, Simonsen JN, Cameron DW, et al. Cofactors in male-female sexual transmission of human immunodeficiency virus type 1. J Infect Dis 1991;163:233-9.

22. Orroth KK, Gavyole A, Todd J, et al. Syndromic treatment of sexually transmitted diseases reduces the proportion of incident HIV infections attributable to these diseases in rural Tanzania. AIDS 2000;14:1429-37.

23. Kaul R, Kimani J, Nagelkerke NJ, et al. Monthly antibiotic chemoprophylaxis and incidence of sexually transmitted infections and HIV-1 infection in Kenyan sex workers: a randomized controlled trial. JAMA 2004;291:2555-62.

24. Sangani $\mathbf{P}$, Rutherford G, Wilkinson D. Population-based interventions for reducing sexually transmitted infections, including HIV infection. Cochrane Database Syst Rev 2004;(2):CD001220. doi:10.1002/14651858.CD001220.pub2

25. Mishra S, Moses S, Hanumaiah PK, et al. Sex work, syphilis, and seeking treatment: an opportunity for intervention in HIV prevention programming in Karnataka, south India. Sex Transm Dis 2009:36:157-64.
26. Lau JT, Wang $\mathrm{R}$, Chen $\mathrm{H}$, et al. Evaluation of the overall program effectiveness of HIV related intervention programs in a community in Sichuan, China. Sex Transm Dis 2007:34:653-62

27. Evans $\mathbf{C}$, Lambert $\mathrm{H}$. The limits of behaviour change theory: condom use and contexts of HIV risk in the Kolkata sex industry. Cult Health Sex 2008;10:27-42.

28. Morris M, Pramualratana A, Podhisita C, et al. The relational determinants of condom use with commercial sex partners in Thailand. AIDS 1995:9:507-15.

29. Cornish F, Ghosh R. The necessary contradictions of 'community-led' health promotion: a case study of HIV prevention in an Indian red light district. Soc Sci Med 2007:64:496-507.

30. National AIDS Control Organisation (NACO). HIV Fact Sheets. Based on HIV Sentinel Surveillance Data in India 2003-2006. New Delhi: NACO, 2007: 59-60. https://www.nacoonline.org/upload/NACO\%20PDF/HIV_Fact_Sheets_2006. pdf (accessed 7 April 2009)

31. Hanck SE, Blankenship KM, Irwin KS, et al. Assessment of self-reported sexual behavior and condom use among female sex workers in India using a polling box approach: a preliminary report. Sex Transm Dis 2008;35:489-94. 